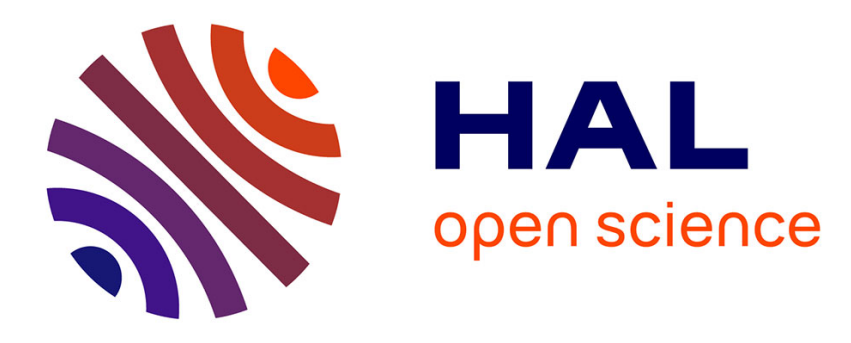

\title{
Absence of proof for the Hohenberg-Kohn theorem for a Hamiltonian linear in the magnetic field
}

\author{
Andreas Savin
}

\section{To cite this version:}

Andreas Savin. Absence of proof for the Hohenberg-Kohn theorem for a Hamiltonian linear in the magnetic field. Molecular Physics, 2016, 10.1080/00268976.2016.1171409 . hal-01317662

\section{HAL Id: hal-01317662 \\ https://hal.sorbonne-universite.fr/hal-01317662}

Submitted on 18 May 2016

HAL is a multi-disciplinary open access archive for the deposit and dissemination of scientific research documents, whether they are published or not. The documents may come from teaching and research institutions in France or abroad, or from public or private research centers.
L'archive ouverte pluridisciplinaire HAL, est destinée au dépôt et à la diffusion de documents scientifiques de niveau recherche, publiés ou non, émanant des établissements d'enseignement et de recherche français ou étrangers, des laboratoires publics ou privés. 


\title{
Absence of proof for the Hohenberg-Kohn theorem for a Hamiltonian linear in the magnetic field *
}

\author{
Andreas Savin ${ }^{1,2}$ \\ ${ }^{1}$ CNRS, UMR7616, Laboratoire de Chimie Théorique, F-75005 \\ Paris, France \\ ${ }^{2}$ UPMC Univ Paris 06, UMR7616, Laboratoire de Chimie \\ Théorique, F-75005 Paris, France
}

May 18, 2016

\begin{abstract}
The wide-spread idea that spin-density functional theory is based upon the extension of the Hohenberg-Kohn theorem to weak magnetic fields is contested. First, it is assumed that only the term linear in magnetic field can be kept in the Hamiltonian. Second, once this is done, two problems arise

i) not only the spin-dependent, but also the orbital-dependent term should be taken care of, and

ii) the latter produces eigenvalues that are not bounded from below, thus invalidating the proof of the Hohenberg-Kohn theorem.
\end{abstract}

\section{Introduction}

The success of density functional theory is largely due to its extension to spinpolarized systems: spin-density functional theory "is much more amenable to semilocal approximation than density functional theory" [1]. In textbooks, the Hohenberg-Kohn theorem is extended for spin polarized systems as

$$
E_{0}=\min _{\rho, m} F[\rho, m]+\int \rho(r) v(r) d^{3} r+\mu_{0} \int B(r) m(r) d^{3} r
$$

Here, $E_{0}$ is the ground state energy, $\rho(r)$ the ground state density, the sum of the up- $\left(\rho_{\uparrow}\right)$ and down-spin density $\left(\rho_{\downarrow}\right), m(r)=\rho_{\uparrow}(r)-\rho_{\downarrow}(r), v(r)$ a potential describing the electrostatic interactions of the electrons with an external (usually Coulomb) field, and $B(r)$ a magnetic field, $\mu_{0}$ the Bohr magneton, and $F$ is a

${ }^{*}$ This paper is dedicated to Hans Jørgen Aagaard Jensen, in continuation to our enjoyable discussions on the meaning of spin-density functional theory, and its extension to multiconfiguration methods. 
functional that depends only on $\rho$ and $m$, but not on $v$ and $B$. To obtain this, the proof of the Hohenberg-Kohn theorem for $B=0$ is repeated step by step. One starts with a Hamiltonian that contains a term linear in the magnetic field, additionally to the usual ones: kinetic energy, interaction between electrons, and between the electrons and the external (usually nuclear) field. Quadratic terms in $B$ should be present, but one argues that they are irrelevant at small $B$. There is also a practical argument for neglecting them: theory would become more complicated, as the current density would be needed, too. To derive the Hohenberg-Kohn theorem in small magnetic fields, one makes use of the variation principle that states that for any wave function the expectation value of the Hamiltonian is larger than $E_{0}$.

The aim of this paper is to show that

- the orbital contribution cannot be neglected, even for small magnetic fields,

- in the presence of a vanishingly small, but non-zero $B$, an infinity of states that are above the ground state in the absence of the magnetic field get below it when the magnetic field is switched on; in fact, the ground state of the Hamiltonian with a term linar in $B$ becomes unbounded from below,

and thus invalidates the extension of the Hohenberg-Kohn theorem that relies on the variation principle. The argumentation given below follows the example given in the classic book of F. Rellich, section "A Term of Second Order in the Perturbation Parameter cannot always be neglected in Comparison with the First Order Term" [2]. As in this book, the hydrogen atom will be considered to exemplify the issue.

\section{Even for weak magnetic fields, orbital contri- bution should not be neglected}

According to the textbook quantum mechanical treatment of the Zeeman effect, (e.g., [3]), i.e., taking into account terms linear in the magnetic field, the Hamiltonian is given by:

$$
\hat{H}(B)=\hat{H}(B=0)+\mu_{0}(\hat{L}+2 \hat{S}) \cdot B
$$

Please notice that both $\hat{L}$ and $\hat{S}$ commute with $\hat{H}$, and thus the eigenfunctions can be chosen not to change with the constant field $B$, while the eigenvalues do change with $B$. The maximum stabilization by the magnetic field is $-\mu_{o}(L+$ $2 S) B$, and because $L$ can take any integer value, its contribution can be much larger than that of $S$. Thus, we consider below the inclusion of the orbital term, due to $L$.

\section{There is no lower bound to the energy for the Hamiltonian linear in $B$}

Using the Hamiltonian of equation 2, the degenerate states for quantum number $n$ have the maximal energy lowering due to the magnetic field given by $-\mu_{0} n B$. 
Its energy is:

$$
E(n, B)=-\frac{1}{2 n^{2}}-\mu_{0} n B
$$
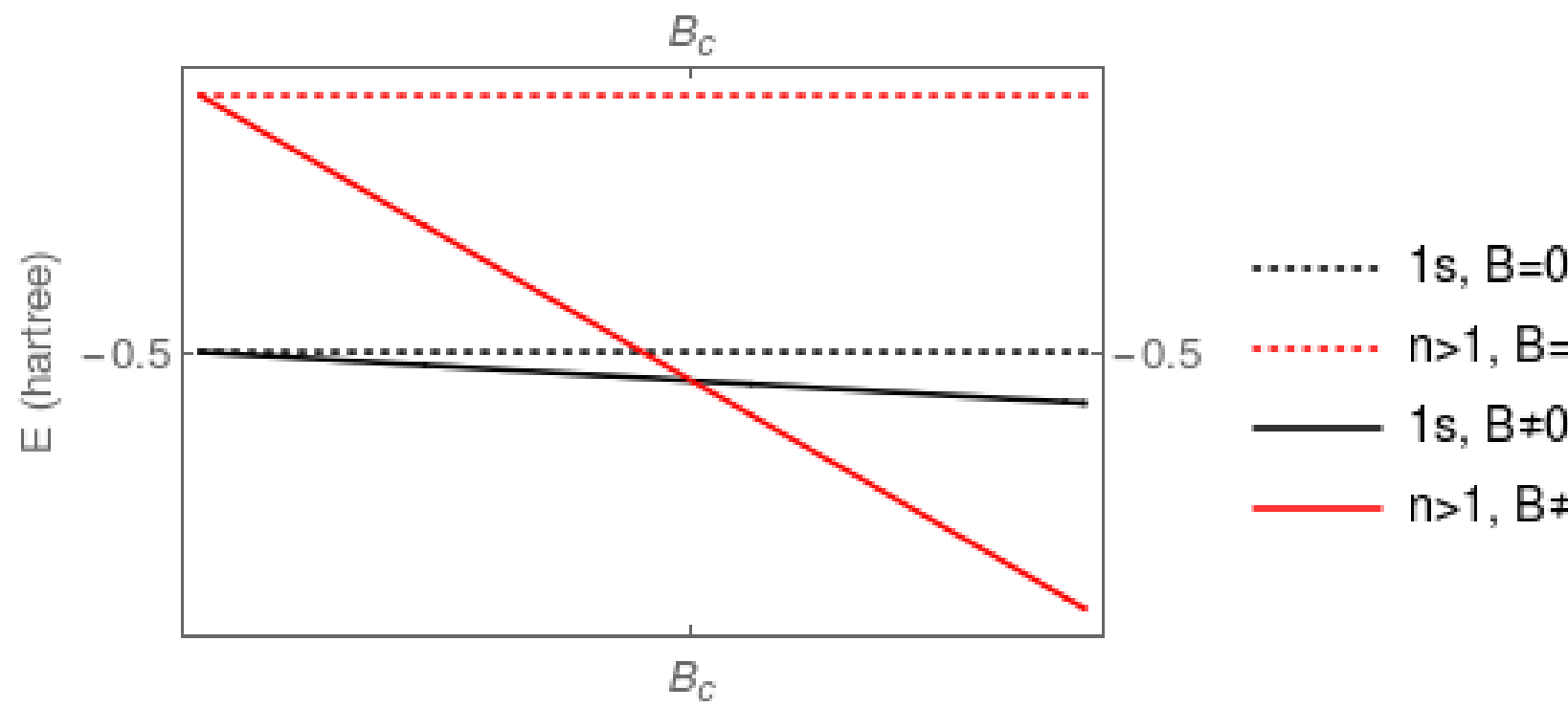

\section{$\mathrm{B}$ (arbitrary units)}

Figure 1: Schematic plot of lowest energies of the hydrogen atomic levels, when only the term linear in the magnetic field is retained, as a function of the magnetic field, $E(n, B)$, for the states having the principal quantum number $n=1$, and $n>1$. The dotted horizontal lines correspond to the energies in absence of the magnetic field. For magnetic fields stronger than $B_{c}, E(n>1, B)$ is lower than that of $E(n=1, B)$.

Figure 1 shows schematically the behavior of $E(n, B)$ as a function of $B$, for two values of $n: n=1$, connected to the 1 s ground state in the absence of magnetic field, and some large value of $n$ : beyond a certain value of the field, $B_{c}$, $E(n>1, B)<E(n=1, B)$ : the stabilization by the magnetic field overcomes the difference between the energies at zero field.

Inversely, we can fix $B$ to some value, and ask if there are values of $n$ for which $E(n, B)<E(n=1, B)$. The behavior of $E(n, B)$ at fixed $B$, is schematically shown in figure 2. At large $n, E(n \gg 1, B)-E\left(n=1, B_{c}\right)$ is dominated by the term that decreases linearly with $n$. There is a critical $n, n_{c}$, beyond which all states have an energy lower than that of the state corresponding to the $1 \mathrm{~s}$ ground state in the absence of the field. As $n$ can take any integer value, there is an infinity of states with $n>n_{c}$. Furthermore, as $n$ increases, $E\left(n, B_{c}\right)$ goes to $-\infty$.

In the argumentation above, no assumption was made about the magnitude of the field, and holds for any non-zero value, no matter how small. It follows that for the form of the Hamiltonian given by equation 2, and any non-zero magnetic field, not only the state corresponding to the ground state at $B=0$ does not remain the ground state, but also that there is now lower bound to the 


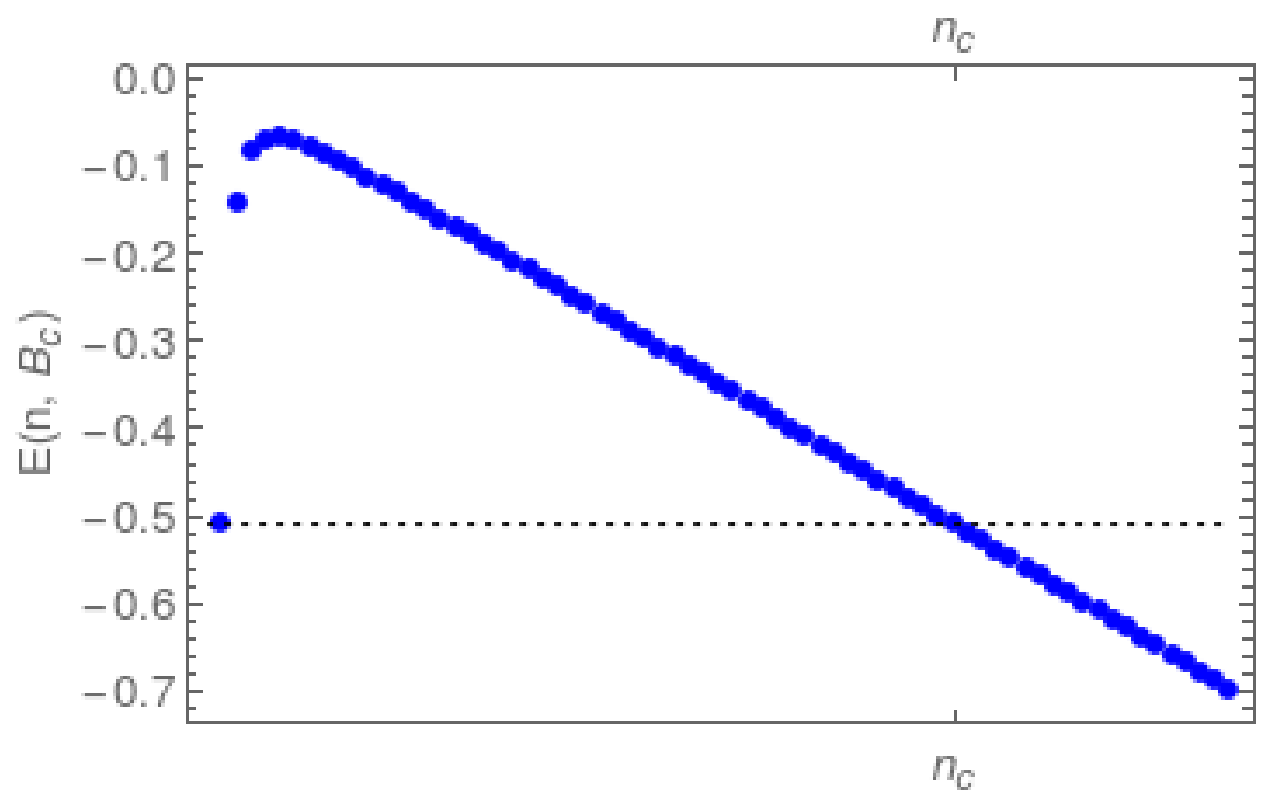

n

Figure 2: Schematic plot of the energies of the hydrogen atomic levels, when only the terms linear in the magnetic field are retained, as a function of the principal quantum number $n$, for a given magnetic field. The states with $n>n_{c}$ have lower energies than that with $n=1$.

energy. Consequently, the variation principle used in deriving the HohenbergKohn theorem cannot be applied for this form of Hamiltonian.

\section{Conclusion}

Using the Hohenberg-Kohn theorem when only linear terms in the magnetic field is questionable because terms originating from $\hat{L} \cdot B$ can become more important than those originating from $\hat{S} \cdot B$. These terms produce energies that are not bounded from below, and the Hohenberg-Kohn theorem derivation for Hamiltonians linear in $B$, equation 2 , is not valid.

This does not mean that the Hohenberg-Kohn cannot be applied, under certain conditions, when a magnetic field is present (see, e.g., [4]). It only means that the Hamiltonian linear in the magnetic field is not suited for it; the quadratic terms, with all the complications they bring, have to be taken into account for a proper derivation of the Hohenberg-Kohn theorem when magnetic fields are present.

The question of the meaning of spin-density functional theory, as largely used nowadays, derived from a Hamiltonian linear in the magnetic field, remains open. 


\section{References}

[1] A. J. Garza, G. E. Scuseria, A. Ruzsinszky, J. Sun, J. P. Perdew, Mol. Phys., DOI: $10.1080 / 00268976.2015 .1114165$

[2] F. Rellich, Perturbation Theory of Eigenvalue Problems, Gorden and Breach, New York, 1969, page 11.

[3] L. D. Landau, E. M. Lifshitz, Quantum Mechanics - Non-relativistic Theory, A Course of Theretical Physics, vol. 3, Pergamon Press, Oxford, 1965, section 112 .

[4] E. I. Tellgren, S. Kvaal, E. Sagvolden, U. Ekström, A. M. Teale, T. Helgaker, Phys. Rev. A 86, 062506 (2012). 